\title{
The Effect of Atmospheric Resistance, Magnetic Force and Oblateness of the Earth on the Motion and Stability of two satellites connected by an extensible cable in circular orbit of the Centre of Mass.
}

\author{
Vijay Kumar ${ }^{1}$, Nikky Kumari ${ }^{2}$ \\ ${ }^{I}$ (Dept. of Mathematics, Mangalayatan University, Beswan, Aligarh, India) \\ ${ }_{2}^{2}$ (Research Scholar, Dept. of Mathematics, Dayalbagh Educational Institute, Agra, India)
}

\begin{abstract}
In a linear motion of a system of two satellites connected by extensible cable, one stable equilibrium point exists when perturbative forces like Solar radiation pressure, shadow of the earth, oblateness of the earth, air resistance and earth's magnetic force act simultaneously. We have obtained one stable point of equilibrium in case of perturbative forces like atmospheric resistance, Magnetic Force and oblateness of the earth acting together on the motion of a system of two satellites connected by extensible cable in the central gravitational field of earth in case of circular orbit of the centre of mass. We have used Liapunov's theorem on stability to examine the stability of the equilibrium point.
\end{abstract}

Keywords: Stability, Equiliribium Point, circular orbit, Liapunov Theorem, Satellites, perturbative forces.

\section{Introduction}

The present paper is concerned with the stability of the equilibrium point of the centre of mass of a system of two satellites connected by a light, flexible and extensible cable under the influence of Atmospheric Resistance, Magnetic Force and oblateness of the earth in case of circular orbit). Beletsky, V.V is the pioneer worker in this field. This paper is an attempt towards the generalization of works done by him.

\section{Equation of motion of the system}

The equations of motion of one of the two satellites moving along Keplerian elliptic orbit under the influence Atmospheric Resistance, Magnetic Force and oblateness of the earth in Nechvill's coordinates system may be obtained by exploiting Lagrange's equations of motion of first kind in the form:

$$
\begin{aligned}
& x^{\prime \prime}-2 y^{\prime}+3 x \rho+\frac{4 A}{\rho}+f \rho \rho^{\prime}+\frac{B}{\rho} \cos i=-\bar{\lambda}_{\alpha}\left[1-\frac{l_{0}}{\rho_{r}}\right] x \rho^{4} \\
& y^{\prime \prime}+2 x^{\prime}+f \rho^{2}-\frac{A}{\rho} y+\frac{B \rho^{\prime}}{\rho^{2}} \cos i=-\overline{\lambda_{\alpha}} \rho^{4}\left[1-\frac{l_{0}}{\rho_{r}}\right] y \\
& \text { and } z^{\prime \prime}+z-\frac{A}{\rho} z=-\overline{\lambda_{\alpha}} \rho^{4}\left[1-\frac{l_{0}}{\rho_{r}}\right] z-\frac{B}{\rho}\left[\frac{\rho^{\prime}}{\rho} \cos (v+w)+\frac{f}{\mu_{E}}\left(3 p^{3} \rho^{2}-\mu_{E}\right) \sin (v+w)\right] \sin i
\end{aligned}
$$

Equation of constraint is given by

$$
x^{2}+y^{2}+z^{2} \leq \frac{l_{0}^{2}}{\rho^{2}}
$$

Where,

$$
r=\sqrt{x^{2}+y^{2}+z^{2}}, A=-\frac{3 k_{2}}{p^{2}}, B=\left(\frac{Q_{1}}{m_{1}}-\frac{Q_{2}}{m_{2}}\right) \frac{\mu_{E}}{\sqrt{\mu p}}, f=\frac{a p^{3}}{\sqrt{\mu p}} \& \overline{\lambda_{\alpha}}=\frac{p^{3}}{\mu} \lambda_{\alpha}=\frac{p^{3}}{\mu}\left(\frac{m_{1}+m_{2}}{m_{1} m_{2} l_{0}}\right) \lambda
$$

For the circular orbit of the centre of mass of the system, we must have

$$
e=0, \rho=\frac{1}{1+e \cos v}=1 \& \rho^{\prime}=0
$$

On putting $\rho=1, \rho^{\prime}=0 \& i=0$ in the equation of motion (1), we get the new system of equation in the form 


$$
\begin{aligned}
& x^{\prime \prime}-2 y^{\prime}-(3-4 A) x+B=-\bar{\lambda}_{\alpha}\left[1-\frac{l_{0}}{r}\right] x \\
& y^{\prime \prime}+2 x^{\prime}+f-A y=-\bar{\lambda}_{\alpha}\left[1-\frac{l_{0}}{r}\right] y \\
& \& z^{\prime \prime}+\mathrm{z}-\mathrm{Az}=-\bar{\lambda}_{\alpha}\left[1-\frac{l_{0}}{r}\right] z
\end{aligned}
$$

The condition of constraints takes the form

$$
x^{2}+y^{2}+z^{2} \leq l_{0}^{2}
$$

Multiplying $1^{\text {st }}, 2^{\text {nd }}$ and $3^{\text {rd }}$ equations of (3) by $2 x^{\prime}, 2 y^{\prime}$ and $2 z^{\prime}$ respectively and adding we get

$$
\left.\begin{array}{l}
2 x^{\prime} x^{\prime \prime}+2 y^{\prime} y^{\prime \prime}+2 z^{\prime} z^{\prime \prime}-(3-4 A) 2 x x^{\prime}+2 B x^{\prime}+2 f y^{\prime}-2 A y y^{\prime}+2(1-A) z z^{\prime} \\
+\lambda \bar{\alpha}\left(2 x x^{\prime}+2 y y^{\prime}+2 z z^{\prime}\right)+\bar{\lambda}_{\alpha} l_{0} \frac{\left(2 x x^{\prime}+2 y y^{\prime}+2 z z^{\prime}\right)}{\sqrt{x^{2}+y^{2}+z^{2}}}=0
\end{array}\right\}
$$

Integrating (2.5) we get Jacobian integral in the form,

$$
x^{\prime 2}+y^{\prime 2}+z^{\prime 2}-(3-4 A) x^{2}+2 B x+2 f y-A y^{2}+(1-A) z^{2}+\bar{\lambda}_{\alpha}\left(x^{2}+y^{2}+z^{2}\right)+\bar{\lambda}_{\alpha} l_{0}\left(x^{2}+y^{2}+z^{2}\right)^{\frac{1}{2}}=h
$$

Where $\mathrm{h}$ is the constant of integration

\section{Equilibrium solution of the problem}

The equilibrium position of the system is given by the constant values of the co-ordinates in rotating frame of references.

Let,

$$
\begin{aligned}
& x=x_{0}, y=y_{0}, z=z_{0} \\
\therefore \quad & x^{\prime}=x_{0}{ }^{\prime}=0=x^{\prime \prime} \\
& y^{\prime}=y_{0}{ }^{\prime}=0=y^{\prime \prime} \\
& z^{\prime}=z_{0}{ }^{\prime}=0=z^{\prime \prime}
\end{aligned}
$$

Where $x_{0}, y_{0}$ and $z_{0}$ are constants give equilibrium position of the system .

Using (3.1) and (3.2) in (2.3) we get

$$
\left.\begin{array}{l}
-(3-4 A) x_{0}+B=-\bar{\lambda}_{\alpha}\left(1-\frac{l_{0}}{r_{0}}\right) x_{0} \\
f-A y_{0}=-\bar{\lambda}_{\alpha}\left(1-\frac{l_{0}}{r_{0}}\right) y_{0} \\
(1-A) z_{0}=-\bar{\lambda}_{\alpha}\left(1-\frac{l_{0}}{r_{0}}\right) z_{0}
\end{array}\right\}
$$

where, $r_{0}=\sqrt{x_{0}^{2}+y_{0}^{2}+z_{0}^{2}}$

Now, we shall discuss the particular solutions of the system of equations (3.3) as follows:

From first and second equations of (3.3) it follows that magnetic force parameter $\mathrm{B}$ and atmospheric resistance parameter $\mathrm{f}$ vanish if $x_{0}=0$ and $y_{0}=0$. Hence $x_{0} \neq 0$ and $y_{0} \neq 0$. If we put $\mathrm{z}_{0}=0$ in the third equations of (3.3) then it is not possible to find the values of $x_{0}=0$ and $y_{0}=0$ from the first two equations of (3.3) Therefore, possible equilibrium position for the system of equations (2.3) may be taken as (a,b,c) Putting $x_{0}=a$ and $y_{0}=b$ and $z_{0}=c$ in equation (3.3) we get 


$$
\left.\begin{array}{l}
-(3-4 A) a+B=-\bar{\lambda}_{\alpha}\left(1-\frac{l_{0}}{r_{0}}\right) a \\
f-A b=\bar{\lambda}_{\alpha}\left(1-\frac{l_{0}}{r_{0}}\right) b \\
(1-A) c=-\bar{\lambda}_{\alpha}\left(1-\frac{l_{0}}{r_{0}}\right) c
\end{array}\right\}
$$

Where, $r_{0}=\sqrt{a^{2}+b^{2}+c^{2}}$

From the last equation of (3.4) we have

$$
1-A=-\lambda \bar{\alpha}\left(1-\frac{l_{0}}{r_{0}}\right) \text { as }(c \neq 0)
$$

Using (3.5) in the first equation and $2^{\text {nd }}$ equation of (3.4) we get

$$
\left.x_{0}=a=\frac{B}{4-5 A} \text { and } y_{0}=\mathrm{b}=f\right\}
$$

From (3.5) we get

$$
\begin{aligned}
& \frac{\overline{\lambda_{\alpha}} l_{0}}{r_{0}}=\overline{\lambda_{\alpha}}+1-A \\
& \therefore \quad r_{0}=\frac{\overline{\lambda_{\alpha}} l_{0}}{\overline{\lambda_{\alpha}}+1-A} \\
& \text { i.e. } \sqrt{a^{2}+b^{2}+c^{2}}=\frac{\overline{\lambda_{\alpha}} l_{0}}{\overline{\lambda_{\alpha}}+1-A} \\
& \therefore \mathrm{c}= \pm \sqrt{\left(\frac{\overline{\lambda_{\alpha}} l_{0}}{\overline{\lambda_{\alpha}}+1-A}\right)^{2}-\left(\frac{B}{4-5 B}\right)^{2}-f^{2}}
\end{aligned}
$$

Hence the equilibrium position in case of circular orbit of the centre of mass of the system is given by

$$
(a, b, c)=\left\{\frac{B}{4-5 B}, f, \sqrt{\left(\frac{\overline{\lambda \alpha}}{\overline{\lambda \alpha+1-A}}\right)^{2}-\left(\frac{B}{4-5 A-f^{2}}\right)^{2}}\right\}
$$

\section{Stability of the system}

Now, we proceed to test the stability of the equilibrium position given by (3.7).

Let $\eta_{1}, \eta_{2}$ and $\eta_{3}$ denote the small variations in the given equilibrium position. Then we have

$$
\left.\begin{array}{l}
x=a+\eta_{1}, y=b+\eta_{2}, \text { and } z=c+\eta_{3} \\
x^{\prime}=\eta_{1}{ }^{\prime}, y^{\prime}=\eta_{2}{ }^{\prime}, \text { and } z^{\prime}=\eta_{3}{ }^{\prime} \\
x^{\prime \prime}=\eta_{1}{ }^{\prime \prime}, y^{\prime \prime}=\eta_{2}{ }^{\prime \prime}, \text { and } z^{\prime \prime}=\eta_{3}{ }^{\prime \prime}
\end{array}\right\}
$$

Using (4.1) in (2.3) we get the variational equations of motion for the system in the form

$$
\left.\begin{array}{l}
\eta_{1}{ }^{\prime}-2 \eta_{2}{ }^{\prime}-(3-4 A)\left(a+\eta_{1}\right)+\mathrm{B}=-\overline{\lambda \alpha}\left[1-\frac{l_{0}}{r}\right]\left(a+\eta_{2}\right) \\
\eta_{2}{ }^{\prime \prime}+2 \eta_{1}^{\prime}+f-A\left(b+\eta_{2}\right)=-\overline{\lambda \alpha}\left[1-\frac{l_{0}}{r}\right]\left(b+\eta_{2}\right) \\
\eta_{3}{ }^{\prime \prime}+(1-A)\left(c+\eta_{3}\right)=-\overline{\lambda \alpha}\left[1-\frac{l_{0}}{r}\right]\left(c+\eta_{3}\right)
\end{array}\right\}
$$

Where, $r^{2}=\left(a+\eta_{1}\right)^{2}+\left(b+\eta_{2}\right)^{2}+\left(c+\eta_{3}\right)^{2}$ 
As the original equations of motion given by (2.3) admits Jacobi's integral, so the variational equations of motion given by (4.2) will also admit jacobi's integral and can be easily obtained by multiplying the three equations of (4.2) by $2\left(a+\eta_{1}\right), 2\left(b+\eta_{2}\right)$ and $2\left(c+\eta_{3}\right)$ respectively and adding them together and integrating in the form.

$$
\begin{aligned}
& \eta_{1}^{2}+\eta_{2}^{2}+\eta_{3}^{2}-(3-4 A)\left(a+\eta_{1}\right)^{2}+2 f\left(a+\eta_{2}\right)-A\left(b+\eta_{2}\right)^{2}+(1-A)\left(c+\eta_{3}\right)^{2}+2 B\left(a+\eta_{1}\right) \\
& +\bar{\lambda}_{\alpha}\left[\left(a+\eta_{1}\right)^{2}+\left(b+\eta_{2}\right)^{2}+\left(c+\eta_{3}\right)^{2}-2 \bar{\lambda}_{\alpha} l_{0} r\left[1+\left\{2\left(a \eta_{1}+b \eta_{2}+c \eta_{3}\right)+\frac{\eta_{1}^{2}+\eta_{2}^{2}+\eta_{3}^{2}}{r^{2}}\right\}\right]^{\frac{1}{2}}\right]=h
\end{aligned}
$$

Where $h$ is the constant of integration. Equation (4.4) gives us the Jacobi's integral for the variational equations of motion of the system at the equilibrium position (a,b.c)

To examine the stability in the sense of Liapunov, we take Jacobian integral as Liapunov's function $V\left(\eta_{1}{ }^{\prime}, \eta_{2}{ }^{\prime}, \eta_{3}{ }^{\prime}, \eta_{1}, \eta_{2}, \eta_{3}\right)$ and is obtained by expanding the terms in (4.4) as

$$
\begin{aligned}
& V\left(\eta_{1}{ }^{\prime}, \eta_{2}{ }^{\prime}, \eta_{3}{ }^{\prime}, \eta_{1}, \eta_{2}, \eta_{3}\right)=\eta_{1}^{2}+\eta_{2}{ }^{2}+\eta_{3}{ }^{2}+\eta_{1}{ }^{2}\left[\overline{\lambda_{\alpha}}+4 A-B-\frac{\overline{\lambda_{\alpha}} l_{0}}{r}+\frac{\overline{\lambda_{\alpha}} l_{0} a^{2}}{r^{3}}\right]+\eta_{2}{ }^{2}\left[\overline{\lambda_{\alpha}}-A-\frac{\overline{\lambda_{\alpha}} l_{0}}{r}+\frac{\bar{\lambda}_{\alpha} l_{0} b^{2}}{r^{3}}\right]+ \\
& \eta_{3}{ }^{2}\left[\bar{\lambda}_{\alpha}+1-A-\frac{\bar{\lambda}_{\alpha} l_{0}}{r}+\frac{\bar{\lambda}_{0} c^{2}}{r^{3}}\right]+\eta_{1}\left[-2 a(3-4 A)+2 B+2 \bar{\lambda}_{\alpha} a-\frac{2 l_{0} \bar{\lambda}_{\alpha} a}{r}\right]+\eta_{2}\left[2 f-2 A b+2 \overline{\lambda_{\alpha}} b-\frac{2 \bar{\lambda}_{\alpha} l_{0} b}{r}\right]+ \\
& \eta_{3}\left[2(1-A) c+2 \bar{\lambda}_{\alpha} c-\frac{2 \bar{\lambda}_{\alpha} l_{0} c}{r}\right]+2 \eta_{1} \eta_{2}\left[\frac{\bar{\lambda}_{\alpha} l_{0} a b}{r^{3}}\right]+2 \eta_{2} \eta_{3}\left[\frac{\bar{\lambda}_{\alpha} l_{0} b c}{r^{3}}\right]+2 \eta_{3} \eta_{1}\left[\frac{\bar{\lambda}_{\alpha} c a}{r^{3}}\right]+o(3)
\end{aligned}
$$

Where $\mathrm{O}(3)$ stands for the third and higher order terms in the small quantities $\eta_{1}, \eta_{2}$, and $\eta_{3}$.

By Liapunov's theorem on stability it follows that the only criterion for given equilibrium position $(\mathrm{a}, \mathrm{b}, \mathrm{c})$ to be stable is that $v$ defined by (4.5) must be positive definite and for this the following conditions must be satisfied:

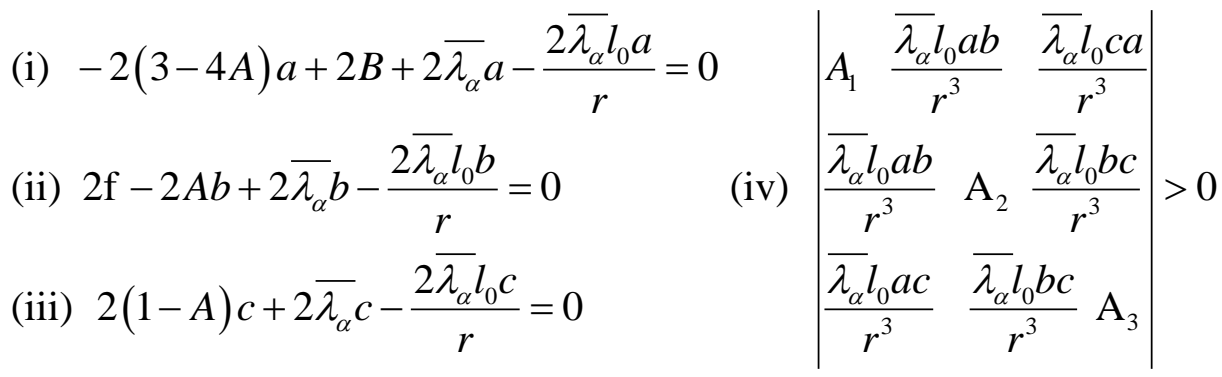

Where,

$$
\left.\begin{array}{l}
A_{1}=\bar{\lambda}_{\alpha}+4 A-3-\frac{\overline{\lambda_{\alpha}} l_{0}}{r}+\frac{\overline{\lambda_{\alpha}} l_{0} a^{2}}{r^{3}} \\
A_{2}=\overline{\lambda_{\alpha}}-A-\frac{\overline{\lambda_{\alpha}} l_{0}}{r}+\frac{\overline{\lambda_{\alpha}} l_{0} b^{2}}{r^{3}} \\
A_{3}=\bar{\lambda}_{\alpha}+1-A-\frac{\bar{\lambda}_{\alpha} l_{0}}{r}+\frac{\overline{\lambda_{\alpha}} l_{0} c^{2}}{r^{3}}
\end{array}\right\}
$$


(v) $\left|\begin{array}{cc}A_{1} & \frac{\bar{\lambda}_{\alpha} l_{0} a b}{r^{3}} \\ \frac{\bar{\lambda}_{\alpha} l_{0} a b}{r^{3}} & \mathrm{~A}_{2}\end{array}\right|>0$

(vi) $\mathrm{A}_{1}>0$

From (4.6), (4.8) and (4.9) we conclude that $A_{1}>0, A_{2}>0$ and $A_{3}>0$. Hence finally the sufficient condition for stability of the equilibrium position $(a, b, c)$ of the system can be put in the form.

(i) $\left[-2 a(3-4 A)+2 B+2 \overline{\lambda_{\alpha}} a-\frac{2 \overline{\lambda_{\alpha}} l_{0} a}{r}\right]=0$
(ii) $\left[2 f-2 A b+2 \overline{\lambda_{\alpha}} b-\frac{2 \overline{\lambda_{\alpha}} l_{0} b}{r}\right]=0$
(iii) $\left[2-(1-A) c+2 \overline{\lambda \alpha} c+\frac{2 \bar{\lambda}_{\alpha} l_{0} c}{r}\right]=0$
(iv) $\left[A_{1}=\overline{\lambda_{\alpha}}+4 A-3-\frac{\overline{\lambda_{\alpha}} l_{0}}{r}+\frac{\overline{\lambda_{\alpha}} l_{0} a^{2}}{r^{3}}\right]>0$
(v) $\left[A_{2}=\overline{\lambda_{\alpha}}-A-\frac{\overline{\lambda_{\alpha}} l_{0}}{r}+\frac{\overline{\lambda_{\alpha}} l_{0} b^{2}}{r^{3}}\right]>0$
(vi) $\left[A_{3}=\overline{\lambda_{\alpha}}+1-A-\frac{\overline{\lambda_{\alpha}} l_{0}}{r}+\frac{\overline{\lambda_{\alpha}} l_{0} c^{2}}{r^{3}}\right]>0$

Now let us proceed to examine the conditions mentioned above in (4.10) one by one to have a clear picture of the stability of the system at the equilibrium point $(\mathrm{a}, \mathrm{b}, \mathrm{c})$

Condition (I):

L.H.S. $=2\left[B-(3-4 A) a+\overline{\lambda \alpha}\left(1-\frac{l_{0}}{r}\right) a\right]=2\left[B-(3-4 A) x_{0}+\overline{\lambda \alpha}\left(1-\frac{l_{0}}{r}\right) x_{0}\right]=2.0=0=$

R.H.S

(II): L.H.S $=2\left[f-A b+\overline{\lambda \alpha}\left(1-\frac{l_{0}}{r}\right) b\right]=2\left[f-A y_{0}+\overline{\lambda \alpha}\left(1-\frac{l_{0}}{r}\right) y_{0}\right]=2.0=0=$ R.H.S

[Using the second equation of (3.4)]

Condition (III)

L.H.S $=2\left[(1-A) c+\overline{\lambda \alpha}\left(1-\frac{l_{0}}{r}\right) c\right]=2\left[(1-A) z_{0}+\overline{\lambda \alpha}\left(1-\frac{l_{0}}{r}\right) z_{0}\right]=2.0=0=$ R.H.S.

Condition (IV)

[Using the third equation of (3.4)] 


$$
\begin{aligned}
& A_{1}=\overline{\lambda_{\alpha}}+4 A-3-\frac{\overline{\lambda_{\alpha}} l_{0}}{r}+\frac{\overline{\lambda_{\alpha}} l_{0} a^{2}}{r^{3}}=\overline{\lambda_{\alpha}}+4 A-3-\frac{\overline{\lambda_{\alpha}} l_{0}\left[\overline{\lambda_{\alpha}}+1-A\right]}{\overline{\lambda_{\alpha}} l_{0}}+\frac{\overline{\lambda_{\alpha}} l_{0}}{r^{3}} a^{2}=5 A-4+\frac{\overline{\lambda_{\alpha}} l_{0}}{r}(a / r)^{2} \\
& =5(A-1)+\frac{1+\overline{\lambda_{\alpha}} l_{0}}{r}(a / r)^{2}=5(A-1)+1+\left(\overline{\lambda_{\alpha}}+1-A\right)(a / r)^{2}>0 \\
& \because r=\frac{\overline{\lambda_{\alpha}} l_{0}}{\bar{\lambda}_{\alpha}+1-A}>0 \Rightarrow \overline{\lambda_{\alpha}}+1-A>0
\end{aligned}
$$

Hence fourth condition is satisfied if A-1 >0

Condition (V):

$$
\begin{aligned}
& A_{2}=\bar{\lambda}_{\alpha}-A-\frac{\bar{\lambda}_{\alpha} l_{0}}{r}+\frac{\bar{\lambda}_{\alpha} l_{0} b^{2}}{r^{3}}=\bar{\lambda}_{\alpha}-A-\frac{\bar{\lambda}_{\alpha} l_{0}\left[\lambda \alpha^{2}+1-A\right]}{\bar{\lambda}_{\alpha} l_{0}}+\frac{\bar{\lambda}_{\alpha} l_{0}}{r}(b / r)^{2}=-1+\frac{\bar{\lambda}_{\alpha} l_{0}}{r} \frac{b^{2}}{r^{2}} \\
& =\left(\bar{\lambda}_{\alpha}+1-A\right) \frac{b^{2}}{r^{2}}-1>0 \\
& \text { if } \frac{r^{2}}{b^{2}}<\bar{\lambda}_{\alpha}+1-A \Rightarrow \frac{1}{b^{2}}<\frac{\bar{\lambda}_{\alpha}+1-A}{r^{2}}
\end{aligned}
$$

Hence fifth condition is satisfied if $\frac{1}{f^{2}}<\frac{\bar{\lambda}_{\alpha}+1-A}{r^{2}}$

Condition (VI)

$$
\begin{aligned}
& A_{3}=\overline{\lambda_{\alpha}}+1-A-\frac{\overline{\lambda_{\alpha}} l_{0}}{r}+\frac{\overline{\lambda_{\alpha}} l_{0} c^{2}}{r^{3}}=\overline{\lambda_{\alpha}}+1-A-\frac{\overline{\lambda_{\alpha}} l_{0}\left[\overline{\lambda_{\alpha}}+1-A\right]}{\overline{\lambda_{\alpha}} l_{0}}+\frac{\overline{\lambda_{\alpha}} l_{0}}{r} \frac{c^{2}}{r^{2}}=\left(\overline{\lambda_{\alpha}}+1-A\right) \frac{c^{2}}{r^{2}}>0 \\
& {\left[\because\left(\overline{\lambda_{\alpha}}+1-A>0, \text { as } r>0\right)\right]}
\end{aligned}
$$

Hence sixth condition is also satisfied. Thus we see that the six conditions of (4.10) for the stability of the equilibrium position $(\mathrm{a}, \mathrm{b}, \mathrm{c})$ are identically satisfied if

$\mathrm{A}>1$ and $-\frac{\sqrt{\lambda_{\alpha}+1-A}}{r}<\frac{1}{b}<\frac{\sqrt{\lambda_{\alpha}+1-A}}{r}$

Hence we conclude that the equilibrium is stable at $(a, b, c)$ in the sense of Liapunov.

\section{Reference:}

[1]. Beletsky, V. V.: About the Relative Motion of Two Connected Bodies in orbit. Kosmicheskiye Issoledovania, vol. 7, No. 6, pp. 827 - 840, 1969 (Russian).

[2]. Thakur ; H.K. : The motion of a system of two satellites connected by extensible cable ; Ph.D. thesis, submitted to B. R. A. Bihar University, Muzaffarpur, 1975

[3]. Singh, R. B.: Three Dimensional motion of system a two cable-connected satellite in orbit. Astronautica, acta, vol. 18, pp. 301 308, 1973

[4]. Singh, A. K. P. : Effect of Earth's Shadow on the motion of a system of two Satellites connected by extensible cable under the influence of solar radiation pressure, Ph. D. Thesis Submitted to B. R. A. Bihar University, Muzaffarpur, 1990.

[5]. Kumar. V and Kamari. N, Stability of the equilibrium point of the Centre of mass of an extensible cable-connected satellites system in case of circular orbit in three dimensional motions, IJSER Volume 4, Issue9, September 2013

[6] Kumar. V and Kumari. N, Effect of the earth's oblateness, the shadow of the earth due to the solar radiation pressure and magnetic force on the motion and stability of two satellites connected by an extensible cable in circular orbit of the centre of mass. IOSR-JM Volume 10, Issue 10, Oct. 2013 